\title{
Partial Pill Counts for Assessments of Medication Adherence in Type 2 Diabetic Patients Treated with Polypharmacy
}

\author{
Mitsuyoshi TAKAHARA*1, Toshihiko SHIRAIWA*1 ${ }^{* 1}$ Naoko OGAWA ${ }^{* 2}$, Mayumi YAMAMOTO*2, \\ Kaoru YAMAMOTO*1 ${ }^{* 1}$ Masayuki DOI ${ }^{* 2}$, Yoko YOSHIDA*1 and Setsuko GOTOU*2
}

\begin{abstract}
Background: Although pill counts can objectively measure medication adherence, they are often labor-intensive and timeconsuming, especially in patients taking numerous medications. We hypothesized that counting pills for not all but only some medications, which would undoubtedly spare time and labor, could provide reliable information on adherence to all medications. The aim of the present study was to evaluate how accurately medication adherence could be assessed using partial pill counts.
\end{abstract}

Methods: We retrospectively analyzed pill count data from 158 consecutive Japanese type 2 diabetic outpatients treated with polypharmacy. Adherence was defined as an $80 \%$ or higher medication consumption rate, whereas non-adherence was defined as $<80 \%$. We assessed how accurately a patient's adherence to one medication could be predicted based on information on his/her adherence to another medication.

Results: The positive likelihood ratio for adherence (i.e., reciprocal of the negative likelihood ratio of non-adherence) was $2.4(95 \%$ confidence interval: 1.7 to 3.3$)$, whereas that for non-adherence (i.e., reciprocal of the negative likelihood ratio of adherence) was 9.9 (95\% confidence interval: 7.1 to 13.8 ) (both $p<0.05)$. The accuracy was dependent on the consistency of administration times. Adherence (or non-adherence) to a medication could be more accurately predicted based on information regarding another medication with the same administration schedule, compared with another with a different administration schedule.

Conclusion: Adherence (or non-adherence) to medications could be predicted with considerable accuracy based on information regarding adherence (or non-adherence) to another medication in type 2 diabetic patients treated with polypharmacy.

Key words: medication adherence, partial pill count, accuracy, polypharmacy

Jpn J Clin Pharmacol Ther. 2017; 48(5) : 173-175

\section{Introduction}

Most type 2 diabetic patients are treated with oral antidiabetic drugs (OADs), and medication adherence is a key factor in the management of type 2 diabetes. ${ }^{1)}$ Monitoring medication adherence in clinical settings is important.

Pill counts are a practical objective measure of medication adherence in clinical settings. ${ }^{2}$ However, these measures are often labor-intensive and time-consuming, especially in patients taking numerous medications. The more medications patients take, the more labor and time are required to count pills. If pill counts can be performed in a less labor-intensive manner, this would be ideal.

One of the possible strategies is to infer medication adherence by counting pills for not all but only some medications; i.e., to count pills for some medications to measure adherence and to infer adherence to other medications by analogy. The strategy would undoubtedly spare labor and time. However, whether or not these partial pill counts would provide clinically reliable information on medication adherence is still unknown.

The aim of the present study was to evaluate how accurately medication adherence could be assessed using partial pill counts in type 2 diabetic patients treated with polypharmacy.

\section{Methods}

A retrospective analysis of pill count data from 158 consecutive Japanese outpatients with type 2 diabetes, who were treated with more than one oral medication, including $\operatorname{OAD}(\mathrm{s})$, and underwent full pill counts in Smile Pharmacy, Osaka, Japan, was performed in the present study. This retrospective study using medical records was conducted in accordance with the Declaration of Helsinki, and was approved by the ethics committee of Shiraiwa Medical Clinic. Informed consent was considered exempt on the grounds that the present study was an observational research using only existing materials; relevant information regarding the study was open to the public in accordance with the Ethical Guidelines for Medical and Health Research Involving Human Subjects in Japan.

\section{Measures of medication adherence}

Adherence to each medication was objectively assessed based on

\footnotetext{
*1 Shiraiwa Medical Clinic, Japan $\quad{ }^{* 2}$ Smile Pharmacy, Japan Address for correspondence: TAKAHARA M. Shiraiwa Medical Clinic, 4-10-24 Hozenji, Kashiwara City, Osaka 582-0005, Japan TEL: +81-72-971-1221 FAX: +81-72-971-1223 E-mail: takahara@endmet.med.osaka-u.ac.jp Manuscript received May 25, 2017; revised June 30, 2017; accepted July 23, 2017

ISSN 0388-1601 Copyright: @2017 the Japanese Society of Clinical Pharmacology and Therapeutics (JSCPT)
} 
pill counts. To perform pill counts, the blister packs of dispensed medications were marked with a pen for the purpose of distinguishing the currently dispensed medications from previously dispensed but unused ones that the patients kept. Patients were asked to take the newly dispensed medications until the next visit, and to leave the old unused ones if there were any. The number of pills taken was calculated as the number of dispensed pills minus the number of remaining pills, and the rate of medication adherence was expressed as a ratio of the number of pills taken to the number of pills that were expected to be taken between two visits. ${ }^{2}$ Adherence was defined as an $80 \%$ or higher medication adherence rate, whereas non-adherence was defined as lower than $80 \%$.

\section{Statistical analysis}

Data are presented as means \pm standard deviation (SD) for continuous variables and as percentages for dichotomous variables. A $p$ value less than 0.05 was considered to be significant, and $95 \%$ confidence intervals are reported when appropriate. We hypothesized that a person's adherence to one medication $\left(M_{\text {tested }}\right)$ would be considerably consistent with his/her adherence to another medication $\left(M_{\text {known }}\right)$, where $M_{\text {tested }}$ denoted an arbitrary medication for which adherence was unknown and $M_{\text {known }}$ denoted another medication for which adherence was known. Sensitivity, specificity, and the likelihood ratio were calculated to assess how accurately a patient's adherence (or nonadherence) to $M_{\text {tested }}$ could be predicted based on the fact that he/ she adhered (or did not adhere) to the administration schedule for $M_{\text {known. }}$ The prevalence of adherence (or non-adherence) was weighted according to the number of medications per patient. For example, if a person treated with five medications adhered to the administration schedule for three of the five medications $(60 \%)$ but did not adhere to the remaining two $(40 \%), 0.6$ persons is regarded as adhering, whereas 0.4 persons did not adhere. Furthermore, the agreement between the adherence (or nonadherence) to one arbitrary medication $M_{\text {tested }}$ and one of the remaining medications $M_{\text {known }}$ can be expressed as a conditional probability. In this example, the combination of $M_{\text {tested }}$ and $M_{\text {known }}$ has ${ }_{5} \mathrm{P}_{2}(=20)$ patterns. Of these, there are ${ }_{3} \mathrm{P}_{2}(=6)$ patterns in which the person adheres to both $M_{\text {tested }}$ and $M_{\text {known }}$, whereas there are ${ }_{2} \mathrm{P}_{2}(=2)$ patterns in which the person does not adhere to both $M_{\text {tested }}$ and $M_{\text {known. }}$. Consequently, $6 / 20(=0.3)$ of the 0.6 persons who adhered to $M_{\text {tested }}$ adhered to $M_{\text {known }}$, and 2/20 $(=0.1)$ of the 0.4 persons who did not adhere to $M_{\text {tested }}$ did not adhere to $M_{\text {known }}$. The sensitivity and specificity of adherence (or non-adherence) in an overall population can be calculated based on this interpretation. The positive likelihood ratio is equivalent to sensitivity/( $1-$ specificity $)$. The confidence intervals of sensitivity and specificity were calculated using the normal approximation to the binomial distribution. The confidence interval and $p$ value of the likelihood ratio were obtained using normal approximation to the logarithmic transformation of the risk ratio. The $p$ value was for the test against 1 for the ratio, i.e., against 0 of the log-transformed ratio.

\section{Results}

The clinical characteristics of the study population are shown in Table 1. A total of 121 patients (77\%) adhered to the administration schedules of all of the medications they took, whereas the
Table 1 Patient characteristics

\begin{tabular}{lc}
\hline$n$ & 158 \\
\hline Age (years) & $64 \pm 11$ \\
\hline Male sex & $91(58 \%)$ \\
\hline Duration of diabetes (years) & $9 \pm 7$ \\
\hline Hemoglobin A1c $(\%)$ & $6.8 \pm 0.8$ \\
\hline Number of oral anti-diabetic medications & $2.3 \pm 0.9$ \\
\hline Number of overall oral medications & $3.9 \pm 1.9$ \\
\hline Pattern of administration schedules ${ }^{*}$ & $2.2 \pm 0.9$ \\
\hline $\begin{array}{l}\text { Data are presented as means } \pm \text { standard deviation or number (percent- } \\
\text { age). }\end{array}$ & \\
$*$ & If a person took some medications before three meals and other \\
medications after breakfast, this was counted as two patterns of \\
administration schedules.
\end{tabular}

remaining $37(23 \%)$ did not adhere to at least one medication. The prevalence of adherence was calculated as $86 \%$ of the overall study population (136.6 per 158 persons).

Table 2 summarizes the accuracy in predicting a patient's adherence to an arbitrary medication $M_{\text {tested }}$ from the information regarding his/her adherence to another medication $M_{\text {known }}$. The positive likelihood ratio of adherence (equal to the reciprocal of the negative likelihood ratio of non-adherence) was 2.4 (95\% confidence interval: 1.7 to 3.3 ), whereas that for nonadherence (equal to the reciprocal of the negative likelihood ratio of adherence) was 9.9 (95\% confidence interval: 7.1 to 13.8) (both $p<0.05$ ). Furthermore, the accuracy increased when $M_{\text {tested }}$ and $M_{\text {known }}$ had the same administration schedule. The information on adherence to a medication with the same administration schedule led to a $3.9(95 \%$ confidence interval: 2.9 to 5.3) positive likelihood ratio of adherence and a 27.3 (95\% confidence interval: 20.1 to 37.0 ) positive likelihood ratio of non-adherence. On the other hand, the information on adherence to a medication with a different administration schedule yielded a $2.0(95 \%$ confidence interval: 1.3 to 3.0$)$ positive likelihood ratio of adherence and a 5.9 (95\% confidence interval: 3.8 to 9.1 ) positive likelihood ratio of nonadherence (Table 2).

\section{Discussion}

The present study revealed that adherence to a medication could be predicted with considerable accuracy based on information on adherence to another medication in type 2 diabetic patients treated with polypharmacy.

Although pill counts can objectively measure medication adherence, ${ }^{2)}$ they are often labor-intensive and time-consuming, especially in patients taking numerous kinds of medications. In the management of type 2 diabetes, combined use of more than one OAD is common. ${ }^{3)}$ In addition, patients with type 2 diabetes are often treated with pharmacotherapy for other lifestyle-related chronic diseases, such as hypertension and dyslipidemia." Consequently, most type 2 diabetic patients are commonly treated with polypharmacy. The process of partial pill counts, i.e., counting pills for not all but only some medications, is expected to save labor and time. 
Table 2 Accuracy in predicting adherence to medications based on information regarding adherence to another medication

\begin{tabular}{|c|c|c|c|c|}
\hline Given information & $\begin{array}{l}\text { Sensitivity of } \\
\text { adherence } \\
\text { (Specificity of } \\
\text { non-adherence) }\end{array}$ & $\begin{array}{l}\text { Specificity of } \\
\text { adherence } \\
\text { (Sensitivity of } \\
\text { non-adherence) }\end{array}$ & $\begin{array}{l}\text { Positive likelihood ratio of } \\
\text { adherence (Reciprocal of } \\
\text { the negative likelihood } \\
\text { ratio of non-adherence) }\end{array}$ & $\begin{array}{l}\text { Positive likelihood ratio of } \\
\text { non-adherence (Reciprocal } \\
\text { of the negative likelihood } \\
\text { ratio of adherence) }\end{array}$ \\
\hline $\begin{array}{l}\text { Adherence to another arbitrary } \\
\text { medication }\end{array}$ & $94 \%(90 \%$ to $98 \%)$ & $61 \%(40 \%$ to $81 \%)$ & $2.4(1.7 \text { to } 3.3)^{*}$ & $9.9(7.1 \text { to } 13.8)^{*}$ \\
\hline $\begin{array}{l}\text { Adherence to another medication } \\
\text { with the same administration } \\
\text { schedule }\end{array}$ & $97 \%(94 \%$ to $100 \%)$ & $75 \%(51 \%$ to $99 \%)$ & $3.9(2.9 \text { to } 5.3)^{*}$ & $27.3(20.1 \text { to } 37.0)^{*}$ \\
\hline $\begin{array}{l}\text { Adherence to another medication } \\
\text { with a different administration } \\
\text { schedule }\end{array}$ & $91 \%(85 \%$ to $96 \%)$ & $54 \%(29 \%$ to $78 \%)$ & $2.0(1.3 \text { to } 3.0)^{*}$ & $5.9(3.8 \text { to } 9.1)^{*}$ \\
\hline
\end{tabular}

Data presented are values $(95 \%$ confidence intervals). Asterisks indicate that likelihood ratios were significantly different from 1.

Our findings indicate that partial pill counts would provide clinically reliable information on medication adherence. In addition, we demonstrated that the accuracy was dependent on the consistency of administration schedules. Adherence to a medication could be more accurately predicted based on adherence to another medication with the same administration schedule, compared with that of a medication with a different administration schedule. Although we did not have data on the times when patients remembered or forgot to take medications, patients are considered likely to take all or none of the medications at a specific time, rather than taking some but not others. Adherence to a medication is therefore likely to be consistent with that of other medications that should be taken at the same time. Our findings indicate that adherence to medications with the same administration schedule could be assessed together by counting pills for one of the medications. These partial pill counts would provide reliable accuracy and would save a considerable amount of time and labor.

The present study population was limited to patients with type 2 diabetes. Further studies would be needed to validate whether or not the current findings would hold true for other populations with chronic diseases.

\section{Conclusion}

Adherence to medications can be predicted with considerable accuracy based on the information regarding adherence to another medication in type 2 diabetic patients treated with polypharmacy.

\section{Funding}

The present study was self-funded.

\section{Conflict of interest statement}

The authors declare that they have no conflicts of interests associated with this manuscript.

\section{References}

1) Ho PM, Rumsfeld JS, Masoudi FA, McClure DL, Plomondon ME, Steiner JF, et al. Effect of medication nonadherence on hospitalization and mortality among patients with diabetes mellitus. Arch Intern Med. 2006; 166 (17) : 1836-41.

2) Lam WY, Fresco P. Medication adherence measures: an overview. Biomed Res Int. 2015; 2015: 217047. doi: 10.1155/2015/217047.

3) Oishi M, Yamazaki K, Okuguchi F, Sugimoto H, Kanatsuka A, Kashiwagi A. Changes in oral antidiabetic prescriptions and improved glycemic control during the years 2002-2011 in Japan (JDDM32). $J$ Diabetes Investig. 2014; 5(5) : 581-7. doi: 10.1111/jdi.12183.

4) Yokoyama H, Oishi M, Takamura H, Yamasaki K, Shirabe SI, Uchida $\mathrm{D}$, et al. Large-scale survey of rates of achieving targets for blood glucose, blood pressure, and lipids and prevalence of complications in type 2 diabetes (JDDM 40). BMJ Open Diabetes Res Care. 2016; 4 (1) : e000294. 